\begin{tabular}{|c|c|}
\hline Title & Topology Optimization Method Based on On-Off Method and Level Set A pproach \\
\hline Author(s) & Hidaka, Y uki; Sato, Takahiro; Igarashi, Haj ime \\
\hline Citation & $\begin{array}{l}\text { IEEE Transactions on Magnetics, 50(2), } 7015204 \\
\text { https://doi.org/10.1109/ MA G.2013.2285379 }\end{array}$ \\
\hline Issue Date & $2014-02$ \\
\hline Doc URL & http://hdl.handle.net/2115/56539 \\
\hline Rights & $\begin{array}{l}\text { (c) } 2014 \text { IEEE. Personal use of this material is permitted. Permission from IEEE must be obtained for all other uses, in } \\
\text { any current or future media, including reprinting/republishing this material for advertising or promotional purposes, } \\
\text { creating new collective works, for resale or redistribution to servers or lists, or reuse of any copyrighted component of } \\
\text { this work in other works. }\end{array}$ \\
\hline Tyре & article (author version) \\
\hline File Information & compumag_hidaka.pdf \\
\hline
\end{tabular}

Instructions for use 


\title{
Topology Optimization Method Based on On-Off Method and Level Set Approach
}

\author{
Yuki Hidaka ${ }^{1}$, Takahiro Sato ${ }^{1}$ and Hajime Igarashi ${ }^{1}$, Member, IEEE \\ ${ }^{1}$ Graduate School of Information Science and Technology, Hokkaido University, \\ Kita 14, Nishi 9, Kita-ku, Sapporo, 060-0814, JAPAN
}

\begin{abstract}
This paper proposes a two-step method for topology optimization, in which after global search is performed by the on-off method, and then local search is carried out by the level set approach. The genetic and gradient-based algorithms are employed for the former and latter optimizations, respectively. The present method is applied to two numerical examples; topology optimizations of a magnetic shield system and IPM-motor. It is shown that the present method can find solutions with better performances in comparison with the conventional on-off method.
\end{abstract}

Index Terms - Genetic algorithms, Level set, Shape optimization, Permanent magnetic motors.

\section{INTRODUCTION}

$\mathrm{S}$ HAPE optimization plays an important role in the development of electromagnetic devices. There are two approaches in the shape optimization, namely, parameter and topology optimizations. In the parameter optimization, device shapes are expressed in terms of geometric parameters and optimization is conducted by changing them [1]-[2]. On the other hand, the topology optimization seeks for the optimum solutions directly varying the material shapes without geometric parameters [3]-[5]. For this reason, quite new device shapes could be found by the topology optimization.

In the topology optimization, the on-off and level set methods have been widely used [3]-[7]. In the on-off method, device shapes are expressed as binary pixel images and are optimized by changing the binary status of the pixels. In this method, genetic algorithm (GA) is often employed for the optimization algorithm. Although the GA-based on-off method would have good performance for the global search, the resultant shapes are sometimes so complicated that they are not suitable for engineering realization. The level set method, on the other hand, expresses material boundaries in terms of the differentiable level set functions. Since the search is carried out using gradient-based methods, the level set method can avoid complexity in the resultant shapes [6]-[7]. However, this method would have poor performance in the global search carried out by the gradient-based method.

In this paper, we present a new topology optimization method which combines the on-off method with level set method. In the present method, first, the global search is performed based on the on-off optimization. During the optimization process, spatially high-frequency components are filtered out using a moving-average technique [8]. Then the solutions obtained by the on-off optimization are improved by the level-set-based optimization, which can effectively perform the local search. It is expected that the final solution obtained by the present two-step method has higher fitness in comparison with the solution obtained by the on-off method.

Manuscript received June 21, 2013. Corresponding author: Yuki Hidaka (e-mail: hidaka@em-si.eng.hokudai.ac.jp).

Digital Object Identifier inserted by IEEE
In order to test the present method, in this work, the shapes of a magnetic shielding and the rotor of an Interior Permanent Magnetic motor (IPM-motor) are optimized [9]-[10].

\section{OPTIMIZATION METHOD}

In this paper, we consider shape optimization of magnetic materials in two-dimensional magnetostatic fields, while the present method is valid for quasi-static and wave problems. In order to compute the properties of the magnetic devises, we solve the finite element equation given by

$K \boldsymbol{a}=\boldsymbol{b}$

where $K, \boldsymbol{a}$ and $\boldsymbol{b}$ represent the finite element matrix, unknown vector potentials and source term.

\section{A. Two-Step Optimization}

We here describe the procedure of the present two-step topology optimization method. In the first step, global search is performed using GA-based on-off method. Thanks to the GA-based optimization, the global exploration is performed with little dependence on the initial shapes. Then, the local search is carried out using the level set method starting from the solution obtained by the first step. It is expected that performance of the solution is improved by the local search of the level set method. Although the resultant shapes obtained by the GA-based optimization may be complicated, the level set method would make the shapes more smooth. It is stressed that the topology of the shape is allowed to change during the second optimization process. Outline of the present method is shown in Fig. 1.

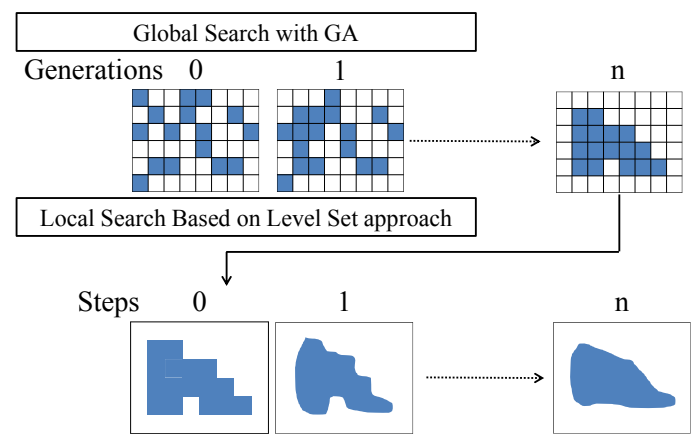

Fig. 1. Outline of the present method. 


\section{B. Global Search Method}

The micro genetic algorithm $(\mu \mathrm{GA})$ [11] is employed for optimization by the on-off method. Since $\mu \mathrm{GA}$ has much smaller populations compared with conventional GA, we can suppress computational time for optimization. If stagnation is observed in the optimization process, the individuals except for elite solution are reinitialized randomly [11]. Procedure of the $\mu \mathrm{GA}$ is summarized as follows:

(1). Initial population is generated randomly which has $N_{\text {pop }}$ size. In this work, population size is set to $N_{\text {pop }}=5$.

(2). Fitness of the each individual are evaluated using Finite Element Method (FEM). The individual which has the highest fitness is selected as elite solution.

(3). Two individuals are selected by performing tournament selection for producing a child by uniform crossover.

(4). $N_{\mathrm{pop}}-1$ individuals are produced by repeating steps 3 .

(5). If the population convergences, the individuals except for elite solution are reinitialized.

(6). Steps 2 to 5 are repeated until the iteration number reaches to maximum.

\section{Local Search Method}

The local search is performed using the level set method. In the level set method, material shape is expressed in terms of the level set function which possesses the following properties

$$
\phi(x)\left\{\begin{array}{cc}
>0 & x \in \Omega \\
=0 & x \in \partial \Omega \\
<0 & x \in D \backslash \Omega
\end{array}\right.
$$

where $D, \Omega$ and $\partial \Omega$ denote design region, material region and material boundary, respectively, and $\boldsymbol{x}$ is a position vector. The material boundaries are expressed with contour line or surfaces where $\phi(x)=0$ is satisfied. The shape optimizations can be performed by changing the distribution of $\phi(x)$. The level set function is constructed using the distance between $\boldsymbol{x}$ and $\partial \Omega$ such that $\phi(x)= \pm \min _{\boldsymbol{y}} d(\boldsymbol{x}, \boldsymbol{y})$, where $\boldsymbol{y} \in \partial \Omega$ and $d$ denotes the Euclidean distance [6].

In order to represent material shapes by the level set function, the magnetic reluctivity $v(\boldsymbol{x})$ is expressed in terms of the sigmoid function as follows:

$$
v(\boldsymbol{x})=v_{0}+\frac{v_{m}-v_{0}}{1+e^{-\phi / \delta}}
$$

where $v_{m}$ and $v_{0}$ denote magnetic reluctivity of core and air. Moreover, $\delta$ is chosen to the element size. In the optimization, the level set function is updated to improve the value of objective function. To express the boundary transition, the hypothetical time, $t$, is introduced. The level set equation, which expresses the transition of the boundaries, is defined by

$$
\frac{\partial \phi(x, t)}{\partial t}+\frac{\mathrm{d} F}{\mathrm{~d} \phi}=0
$$

where $F$ denotes the objective function. Since it is difficult to evaluate $\mathrm{d} F / \mathrm{d} \phi$ analytically, it is computed using the adjoint variable method [6]. That is, $F$ is approximated by the modified objective function $\hat{F}$ defined by $\hat{F}=F+z^{T}(K a-b)$, where $\boldsymbol{a} \approx K^{-1} \boldsymbol{b}$ is the numerical solution to the FE equation of magnetostatic field. The derivative of $\hat{F}$ is now given by

$\frac{\mathrm{d} \hat{F}}{\mathrm{~d} \phi_{i}}=\frac{\partial F}{\partial \phi_{i}}+z^{\mathrm{T}} \frac{\partial K}{\partial \phi_{i}} \boldsymbol{a}$

where $z$ is the solution to $K z=-\partial F / \partial \boldsymbol{a}$. The first term in (5) is evaluated when $F$ is an explicit function of $\phi$ as in the case that $F$ depends on the material area. The second term can be evaluated from (3), that is, entities in $K$ which include (3) are differentiated in terms of $\phi$.

\section{NUMERICAL EXAMPLES}

\section{A. Magnetic Shielding System}

\section{1) Optimization Problem}

The present method is applied to the magnetic shield model shown in Fig. 2 [12]. The purpose of this optimization is to minimize the magnetic flux in the evaluated region and area of core. The objective function is defined by

$$
F_{M}(\phi)=W_{M} \frac{|\boldsymbol{B}|_{\text {average }}}{B_{0}}+\frac{S}{S_{\text {design }}}
$$

where $S, S_{\text {design }}, W_{M}$ and $B_{0}$ denote the area of the core and design region, weighting coefficient and normalization constant which is set to $B_{0}=10^{-5} \mathrm{~T}$. Moreover, $|\boldsymbol{B}|_{\text {average }}$ is the average of magnetic flux density in the evaluated region. The magnetic field is analyzed by solving (1), also in the next example.

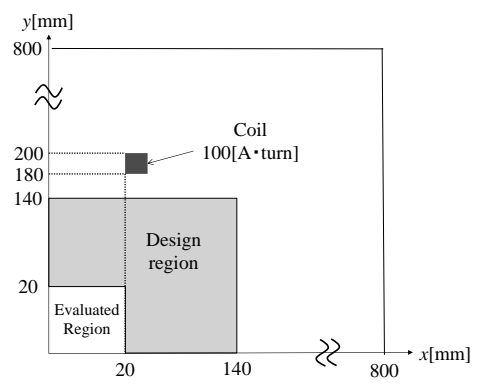

Fig. 2. Magnetic shield model ( $\mu_{r}=200$ in design region).

2) Optimization Result

Optimization parameters are summarized in Table I. The maximum generations and number of steps in the global and local searches are determined so that the optimization processes are sufficiently convergent. The resultant shapes obtained by the present and the conventional on-off methods are shown in Fig. 3. It is notable that the double shielding layers are obtained starting from the uniform design region. The values of flux density, core area and objective function are summarized in Table II. It can be seen from these results that the resultant solution obtained by the present method has the smaller value of objective function than that of the conventional on-off method. Moreover, the material boundary in Fig. 3(b) is found to be more smooth than that in Fig. 3(a).

TABLE I

OPTIMIZATION PARAMETERS

Number of elements in design and analysis regions Max. generation of global search Max. number of steps in local search
2488,5052 200 200 


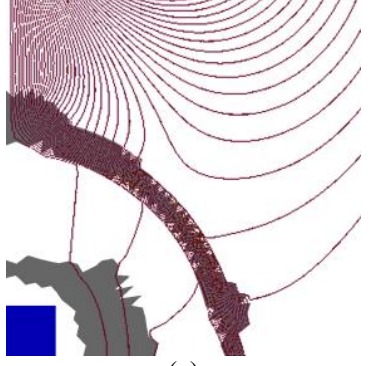

(a)

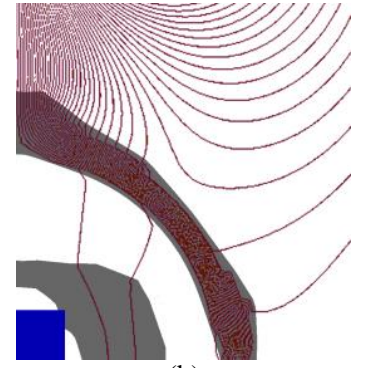

(b)
Fig. 3. Optimized shape and flux distribution on $W_{M}=0.2$. (a) Conventional on-off method. (b) Present method.

TABLE II

OPTIMIZATION RESULT $\left(W_{M}=0.2\right)$

\begin{tabular}{ccc}
\hline \hline & $\begin{array}{c}\text { Conventional on-off } \\
\text { method }\end{array}$ & Present method \\
\hline$|\boldsymbol{B}|_{\text {average }} / B_{0}$ & 0.124 & 0.0990 \\
Area of the core $\left(\mathrm{cm}^{2}\right)$ & 8.230 & 8.309 \\
Objective function & 0.0678 & 0.0631 \\
\hline
\end{tabular}

3) Effect of Weighting Coefficient

In order to consider the influence of the weighting coefficient $W_{M}$ on the results, optimization is performed for different values of $W_{M}$.

In Fig. 4, the resultant values of the first and second terms in (6) are plotted for the conventional on-off and present methods. From these results, it is found that the level set method reduces $|\boldsymbol{B}|_{\text {average }}$, while the area is a little increased. This would be due to the fact that the surface on which $v=v_{\mathrm{m}} / 2$ is satisfied is identified as the material boundary in the level set method, and consequently the mean permeability in the material region is lower than that of the conventional on-off method. The resultant shapes for $W_{M}=0.4$ are shown in Fig. 5 . The swell in the outer shield in Fig. 5 seems to make the flux go far away from the evaluated region.

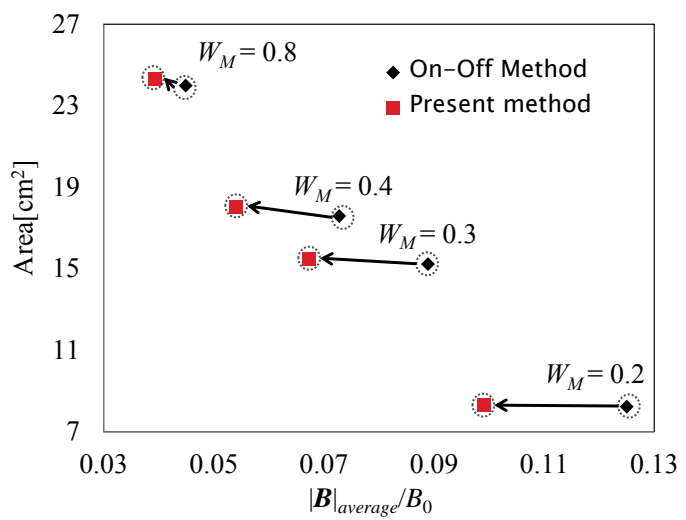

Fig. 4. Comparison of objective function on each weighting coefficient.

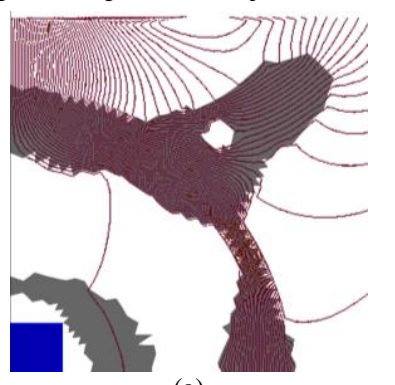

(a)

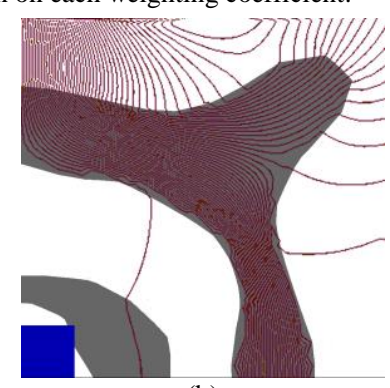

(b)
Fig. 5. Optimized shape on $W_{M}=0.4$. (a) Conventional on-off method. (b) Present method.

\section{B. IPM-Motor}

\section{1) Optimization Problem}

As the second example, we optimize the rotor shape of an IPM-motor shown in Fig. 6(a) [13]. Due to the symmetry, the $1 / 4$ model is analyzed. The purpose of this optimization is to maximize the torque average and minimize the torque ripple of the IPM-motor. The objective function defined by

$F_{I}(\phi)=-W_{I} \frac{T_{\text {average }}}{T_{\text {average }}^{0}}+\frac{T_{\text {ripple }}}{T_{\text {ripple }}^{0}}$

is minimized where $W_{I}$ is a weighting coefficient, $T_{\text {average }}$ and $T_{\text {ripple }}$ are torque average and torque ripple. In (7) the normalization constants are set as $T_{\text {average }}^{0}=3.971 \mathrm{Nm}$ and $T_{\text {ripple }}^{0}=0.353$ which are obtained for the reference model shown in Fig. 6(b). In this work, $T_{\text {ripple }}$ is evaluated by $T_{\text {ripple }}=\left(T_{\max }-T_{\min }\right) / T_{\text {average }}$ where $T_{\max }$ and $T_{\min }$ are the maximum and minimum torques values. In Table III, the analysis conditions and motor specification are summarized.

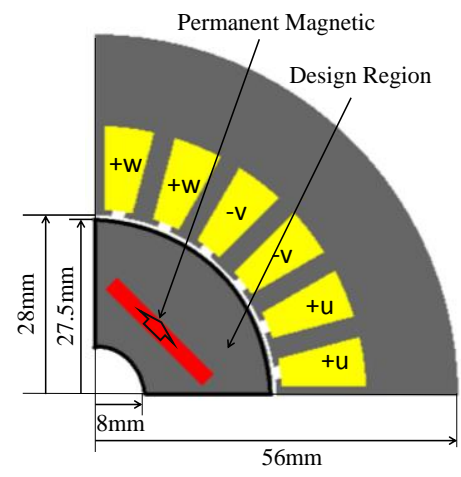

(a)

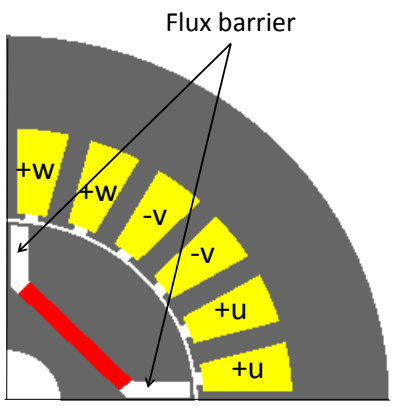

(b)
Fig. 6. Optimization Model. (a) Motor model. (b) Reference model with flux barrier. Nonlinaer BH curve of S45C is assumed for the materials. TABLE III

ANALYSIS CONDITIONS AND SPECIFICATIONS

\begin{tabular}{cc}
\hline Rotation speed (rpm) & 3000 \\
Armature current (A) & 600 \\
Phase of current (degree) & 20 \\
Residual fulx density of PM (T) & 1.0 \\
Width of teeth (mm) & 3.3 \\
Length of Coil (mm) & 25.9 \\
Thickness of PM (mm) & 2.5 \\
Width of PM (mm) & 21 \\
\hline
\end{tabular}

2) Optimization Result

Optimization parameters are summarized in Table. IV. The resultant shapes obtained by the present and conventional onoff methods are shown in Fig. 7. We can see in Fig.7 that the size of the holes near the rotor surface is reduced after the local search. The values of $T_{\text {average }}, T_{\text {ripple }}$, and objective function are summarized in Table. V. It can be seem from these results that both $T_{\text {average }}$ and $T_{\text {ripple }}$ are improved by the level set method. As a result, the solution obtained by the present method has the smaller value of objective function than that of the conventional on-off method. Fig. 8 shows the comparison of torque waves of the two methods.

TABLE IV

OPTIMIZATION PARAMETERS

Number of elements in design and analysis regions

Max. generation of global search

Max. number of steps in local search

1412,5298 100 100 


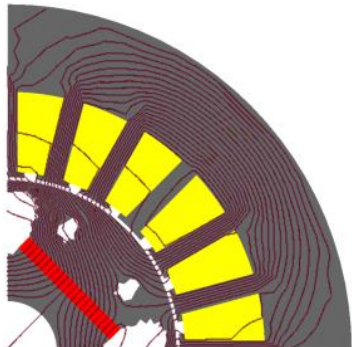

(a)

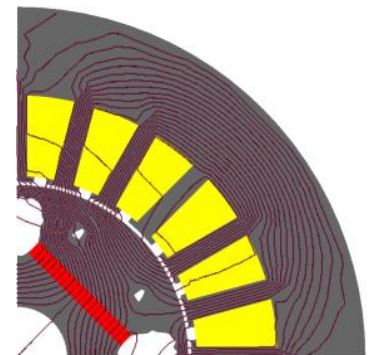

(b)
Fig. 7. Optimized shape on $W_{I}=1.0$. (a) Conventional on-off method. (b) Present method.

TABLE V

OPTIMIZATION RESULT $\left(W_{l}=1.0\right)$

\begin{tabular}{ccc}
\hline \hline & $\begin{array}{c}\text { Conventional on-off } \\
\text { method }\end{array}$ & Present method \\
\hline$T_{\text {average }}(\mathrm{Nm})$ & 5.280 & 5.309 \\
$T_{\text {ripple }}$ & 0.184 & 0.112 \\
Objective function & -0.806 & -1.018 \\
\hline
\end{tabular}

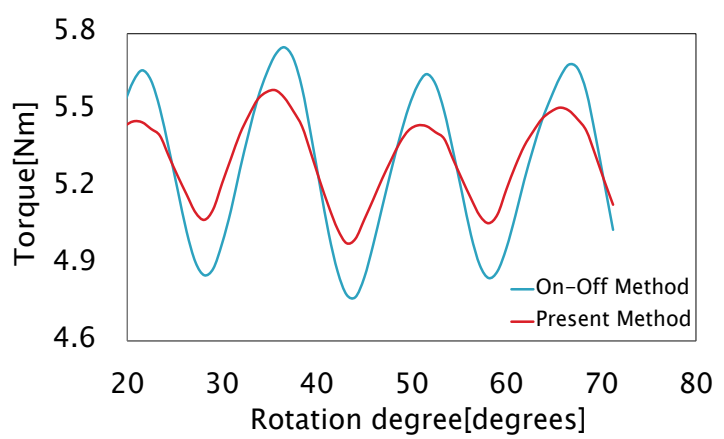

Fig. 8. Comparison of torque $\left(W_{l}=1.0\right)$.

3) Effect of Weighting Coefficient

In order to consider the influence of the weighting coefficient $W_{I}$ on the results, optimization is performed for different values of $W_{I}$.

In Fig. 9, the resultant values of $T_{\text {average }}$ and $T_{\text {ripple }}$ are plotted for the conventional on-off and present methods. From Fig. 9, we can see that the both quantities are improved by the local search of the level set method. The solutions obtained by the present method do not form the Pareto front. This would be attributed to the gradient-based search in the level set method. Fig. 10 shows the shapes of the optimal solutions for $W_{I}=0.3$. The shapes of the flux barrier are found to be much different from those in Fig. 7.

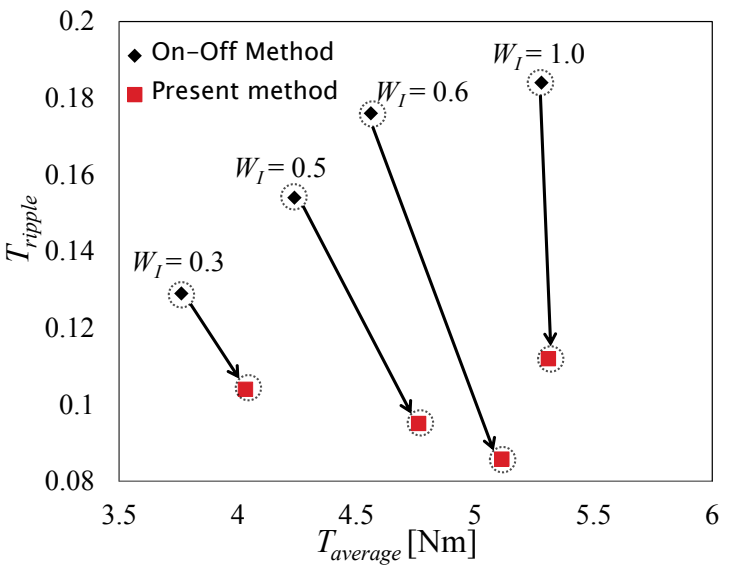

Fig. 9. Comparison of objective function on each weighting coefficient.

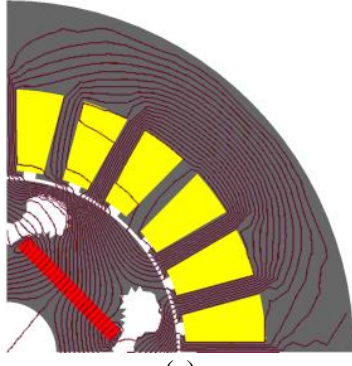

(a)

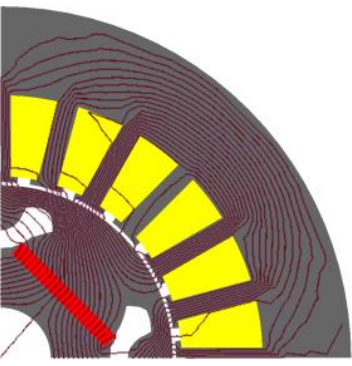

(b)
Fig. 10. Optimized shape on $W_{l}=0.3$. (a) Conventional on-off method. (b) Present method.

\section{CONCLUSION}

In this paper, we have proposed a novel topology optimization method based on on-off and level set methods. In the present method, the optimization process is divided into two steps. At first, the global search is conducted using GA based on-off method. Then, the solution obtained by the global search is improved by the local search using gradient-based level set method. The shapes of the magnetic shielding system and IPM motor have been optimized for test of the present method. We have found that the quality of the optimal solutions is much improved by the present method. For the future work, we plan to apply the present method to three dimensional problems.

\section{REFERENCES}

[1] K. Watanabe, F. Campelo, Y.Iijima, K. Kawano, T. Matsuo, T. Mifune and $\mathrm{H}$. Igarashi, "Optimization of inductors using evolutionary algorithms and its experimental validation," IEEE Trans. Magn., vol. 46, no. 8, pp. 3393-3396, 2010.

[2] J. Cros, P. Viarouge and M. T. Kakhki, "Design and optimization of soft magnetic composite machines with finite element methods," IEEE Trans. Magn., vol. 47, no. 10, pp. 4384-4390, 2011.

[3] J.-K. Byun, I.-H. Park and S.-Y. Hahn, "Topology optimization of electrostatic actuator using design sensitivity," IEEE Trans. Magn., vol. 38, no. 2, pp. 1053-1056, 2002.

[4] D. H. Kim, J. K. Sykulski and D. A. Lowther, "The implications of the use of composite materials in electromagnetic device topology and shape optimization," IEEE Trans. Magn., vol. 45, no. 3, pp. 1154-1157, 2009.

[5] N. Takahashi, T. Yamada, S. Shimose, D. Miyagi, "Optimization of rotor of actual IPM motor using ON/OFF method," IEEE Trans. Magn., vol. 47, no. 5, pp. 1262-1265, 2011.

[6] S. Park, S. Min, S. Yamasaki, S. Nishiwaki and J. Yoo, "Magnetic actuator design using level set based topology optimization," IEEE Trans. Magn., vol. 44, no. 11, pp. 4037-4040, 2008.

[7] Jangwon Lee and Semyung Wang, "Topological shape optimization of permanent magnet in voice coil motor using level set method," IEEE Trans. Magn., vol. 48, no. 2, pp.931-934, 2012.

[8] F. Gustafsson, Adaptive filtering and change detection, John wiley \& sons, LTD, 2000.

[9] D. H. Kim, I. H. Park, J. H. Lee and C. E. Kim, "Optimal shape design of iron core to reduce cogging torque of IPM motor," IEEE Trans. Magn., vol. 39, no. 3, pp. 1456-1459, 2003.

[10] S. -I. Kim et al, "Optimization for reduction of torque ripple in interior permanent magnet motor by using the Tagauchi method," IEEE Trans. Magn., vol. 41, no. 5, pp. 1796-1799, 2005.

[11] C. A. Coello and G. T. Pulido, "A micro-genetic algorithm for multiobjective optimization," EMO 2001, LNCS 1003, pp, 126-140, 2001.

[12] T. Sato, K. Watanabe and H. Igarashi, "A modified immune algorithm with spatial filtering for multi-objective topology optimization of electromagnetic devices," COMPEL, to be published.

[13] Technical report of the institute of electrical engineering of Japan, industry application society, no. 776, 2000. 